\title{
Development an instrument assessing residents' attitude towards professionalism lapses in training
}

\author{
Hyo-Jin Kwon, Young-Mee Lee, Young-Hee Lee and Hyung-Joo Chang \\ Division of Medical Education, Department of Medical Humanities, Korea University College of Medicine, Seoul, \\ Korea
}

Purpose: Medical professionalism is a fundamental competency for all physicians and continuous development of professionalism during residency training is crucial. The purpose of this study was to develop an instrument assessing residents' attitudes toward unprofessional behaviors.

Methods: A questionnaire survey was conducted in cooperation with the Korea Resident Association from May to July 2013. A total of 317 residents from seven university-affiliated hospitals in South Korea participated in the survey.

Results: In the exploratory factor analysis, seven factors were extracted from the data; factor loadings of the 44 items ranged between 0.40 and 0.89 . Through iterative discussion, three items below 0.45 were deleted and one additional item was removed due to its irrelevance. Twelve items included in Factor 1 were divided into two different categories. A final version of the questionnaire containing 40 items in eight categories was assessed using confirmatory factor analysis. It was deemed to have a good fit; the root mean square error of approximation and comparative fit index were 0.07 and 0.9 , respectively. The reliability (Cronbach's $\alpha$ ) of the inventory was 0.97 .

Conclusion: The items of this instrument encompass a broad range of residents' behaviors in clinical practice, research, and publication. In addition, it includes some types of misconduct that can be considered unique features of the authors' cultural backgrounds. We recommend this instrument as an assessment tool to diagnose residents' perceptions and attitudes towards professionalism lapses and to provide insight regarding potential improvement in professionalism education.

Key Words: Professionalism, Professional misconduct, Attitude, Instrumentation

\section{Introduction}

During the last two decades, attention on medical professionalism has increased as a fundamental competence of physicians [1]. In Western countries, various studies have been conducted to understand medical professionalism and embed it in the education field. As a result of previous studies, a concrete concept of medical professionalism has been drawn up, and essential values, principles, and codes of conduct for doctors have been established [2,3,4]. In addition, other previous studies have established a framework and standards for assessing medical professionalism [2,5], which have been employed in evaluating the effects of professionalism education [3,4]. Most medical educators
Received: January 18, 2017 • Revised: March 8, 2017 • Accepted: April 12, 2017 Corresponding Author: Young-Mee Lee (http://orcid.org/0000-0002-4685-9465) Department of Medical Humanities, Korea University College of Medicine, 73 Inchon-ro, Seongbuk-gu, Seoul 02841, Korea

Tel: +82.2.920.6098 Fax: +82.2.928.1647 email: ymleehj@korea.ac.kr
Korean J Med Educ 2017 Jun; 29(2): 81-91.

https://doi.org/10.3946/kjme.2017.55

eISSN: 2005-7288

(C) The Korean Society of Medical Education. All rights reserved. This is an open-access article distributed under the terms of the Creative Commons Attribution Non-Commercial License (http:// creativecommons.org/licenses/by-nc/3.0/), which permits unrestricted non-commercial use, distribution, and reproduction in any medium, provided the original work is properly cited. 
Hyo-Jin Kwon, et al : Development an instrument assessing residents' attitudes towards professionalism lapses

now agree with the importance of evaluating medical professionalism since it induces physicians or students to compensate for their professional defects $[5,6]$.

To evaluate professionalism, the process of identifying unprofessional behavior is necessary as a basic step [7]. Behavioral assessments are proxy measures, resting on the assumption that observed behaviors are reflective of underlying dimensions [3]. Therefore, earlier studies on medical professionalism have focused on the assessment of unprofessional behavior [8]. In particular, many previous studies have paid attention to the professional lapses of residents due to the concern that unprofessional behaviors during training may lead to unprofessional behavior later on when in unsupervised practice $[9,10]$. Evidence for this assumption has come from several studies, which have demonstrated that physicians who have been disciplined for unprofessional behavior during their practice were more likely to have records of irresponsible or unprofessional behavior during medical school years or residency training [9]. Although some current studies have begun to adopt direct evaluation of professional behavior, the assessment of unprofessional behavior is more widely used in the medical education field as the definition of professionalism remains incomplete [8]. However, studies for assessing neither unprofessional behavior, nor direct evaluation of professional behavior have not been widely conducted in Asian cultures.

The residency is a critical period in the formation of professional identity and development of professionalism, and is accompanied by the struggle to operationalize the learning of professional behaviors. Knowing more about residents' perceptions of unprofessional behaviors and the prevalence of lapses in professionalism would enhance definitions and facilitate support for the development of professionalism skills and behaviors at the graduate level $[9,11]$. A valid and reliable instrument to assess unprofessional behaviors can help us to identify program needs and training gaps at both individual and environmental levels.

As a set of values, medical professionalism is inevitably influenced by a certain culture in which it is practiced. Some studies have drawn attention to cultural differences in the conceptualization of medical professionalism in response to varied societal needs [12,13]. An individual's attitude towards unprofessional behavior reflects the context of their cultural and social background. Therefore, we need professionalism assessment tools which can embrace both specific cultural backgrounds of medical practices and core common factors of medical professionalism regardless of difference in culture.

In this study, the authors aimed to develop an inventory assessing residents' attitudes towards unprofessional behaviors which reflects medical professionalism in our own unique medical contexts and cultural backgrounds.

\section{Subjects and methods}

An inventory assessing residents' attitudes toward unprofessional conduct was developed in three steps: questionnaire construction, questionnaire survey, and psychometric analysis of the inventory (Fig. 1).

\section{Development of operational definition of medical professionalism}

As the first step in constructing survey items, an operational definition of medical professionalism was developed through literature review. Professionalism recommendations by the Accreditation Council for Graduate Medical Education (common program requirements), American Board of Internal Medicine (the phy- 
Fig. 1. Development Process of the Instrument to Assess Residents' Attitudes towards Professionalism Lapses

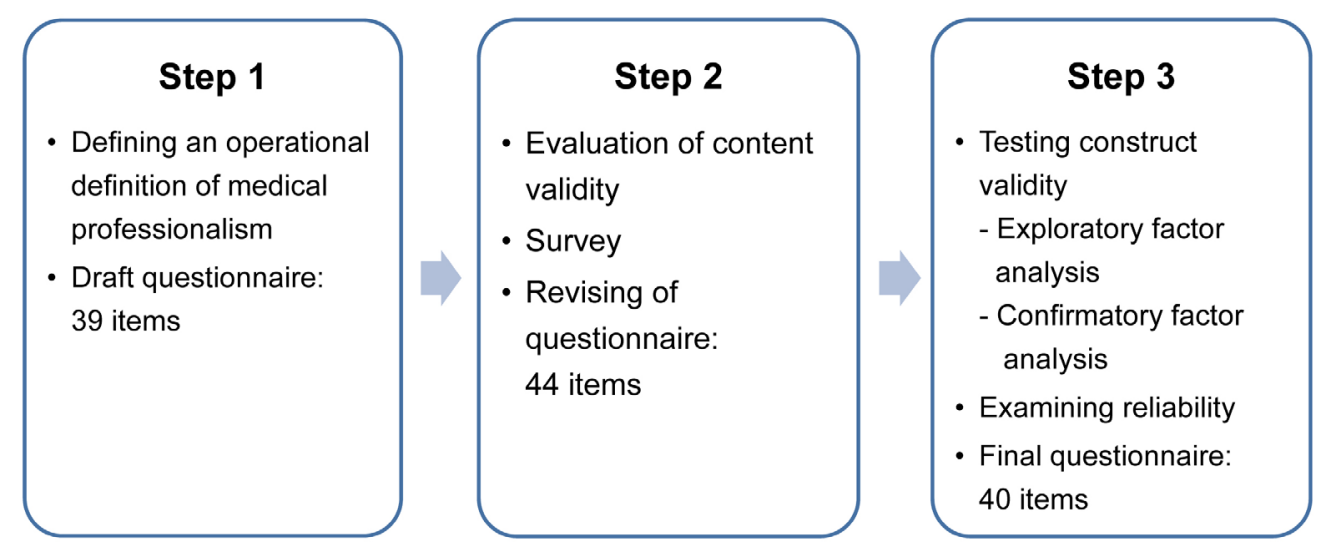

sician charter), as well as definitions of professionalism by the Association of American Medical Colleges and other efforts $[5,14,15,16]$ to establish a professionalism framework were thoroughly reviewed by the authors.

After extracting the core components of medical professionalism from the previous literature, the authors selected those concepts that were repeatedly mentioned and created an operational definition of medical professionalism with the follow domains: (1) clinical competence $[5,14,15]$; (2) commitment to ongoing professional development [5,14]; (3) adherence to ethical standards $[14,15,16]$; (4) promotion of the public good [14,15]; (5) adherence to core human values such as integrity, honesty, altruism, humility, respect for diversity, and transparency with respect to potential conflicts of interest $[5,14,15]$; (6) taking responsibility for their own health and well-being and that of their colleagues [14]; (7) physician-led regulation [5,15]; (8) understanding that physicians are accountable to those served, to society, to their profession, and to themselves $[5,14,15]$; (9) establishing cooperative relations with colleagues and other healthcare professions [5].

\section{The development of a draft questionnaire}

Based upon our operational definition of medical professionalism, in order to develop questionnaire items, the authors focused on common issues closely related with residency training in everyday practice. A draft questionnaire construction was begun under the frame of six domains: (1) clinical competence; (2) commitment to ongoing professional development; (3) adherence to ethical standards; (4) adherence to core human values, particularly integrity and honesty; (5) transparency with respect to potential conflicts of interest; (6) establishing cooperative relations with colleagues and other healthcare professionals.

We searched previous studies on unprofessional behavior by physicians using the keywords "misconduct," "unethical behavior," and "unprofessionalism." Through content analysis of the articles $[7,8,9,10]$, the common descriptors of unprofessional and unethical behaviors during residency training were extracted. To reflect our unique medical culture and actual training environment in the questionnaire items, the types of resident misconduct identified by the authors of previous studies [17] were incorporated into the items. Finally, we selected 39 unprofessional behaviors considered to reflect the authentic and realistic experience in the residency-training environment of Korea.

The questionnaire items consisted of asking residents about their attitudes toward misconduct under the leading question: What do you think of residents' 
Hyo-Jin Kwon, et al : Development an instrument assessing residents' attitudes towards professionalism lapses

conduct with regard to each of the following types of behavior? Please select one answer that best reflects your opinion or views. The answer options consisted of a 4-point Likert scale from 1 (absolutely cannot be done), 2 (cannot be done), 3 (can be done depending on circumstances), to 4 (usually can be done).

\section{Modification of questionnaire items by content validity assessment}

Content validity was assessed by seven panelists: two residents (third- and fourth-year), three clinical faculty members (internal medicine, surgery, and pathology), and two experts in educational measurement. The residents and clinical faculty members focused on evaluating the appropriateness and authenticity of the described clinical situations and misconduct, and the clarity of expression in the questionnaire. The experts in educational measurement mainly assessed the appropriateness of the Likert scale for the response options. Following the expert panel's suggestions, minor amendments were made: (1) the addition of five items which were commonly occurred in real clinical settings of South Korea (providing incorrect information to patients to conceal one's ignorance; prescribing patients with unnecessary medication for doctor's benefit; not reporting patient's medical condition to supervisor to avoid blame; ignoring opinion of other healthcare professionals; criticizing colleague doctors or other healthcare professionals in front of patients), (2) sequencing items according to the relevance of contents, and (3) arranging items in order of the degree of severity of misconduct. As a result, a 44-item questionnaire was developed.

\section{Survey}

A survey using the 44-item questionnaire was conducted in cooperation with the Korea Resident Association from May to July 2013. The Korea Resident
Association consists of resident representatives from training hospitals nationwide. Among the resident representatives, seven members volunteered to help with this survey, all of whom were from university-affiliated hospitals located in various regions of South Korea: Seoul (four hospitals), Goyang (one hospital), Suwon (one hospital), and Daejeon (one hospital). They helped us to recruit participants, and distributed and collected the survey forms. A small gratuity (US \$90) was given to the resident representatives. All participation occurred on a voluntary basis after obtaining informed consent. Residents who agreed to participate in the study were asked to respond to the questionnaire. The data were collected anonymously, and no personal identifiers were used. A total of 317 residents completed the questionnaire.

\section{The assessment of construct validity and reliability}

We examined the construct validity of the questionnaire by factor analysis. Since prior knowledge of residents' attitudes toward unprofessional conduct is lacking, we extracted factors by exploratory factor analysis and then verified the structure of variables by confirmatory factor analysis. A principal component and varimax rotation was used for the exploratory factor analysis.

In the confirmatory factor analysis of this study, two goodness-of-fit indices were used to evaluate the structural equation model. The first index was the root mean square error of approximation (RMSEA), which is an absolute fit measure that takes into account both structural error and parsimony, and which is not affected by sample size. The second was a comparative fit index (CFI), which is based on an assumption that is more realistic and that is also not affected by sample size.

The coefficient of internal consistency reliability was 
assessed by Cronbach's $\alpha$. For statistical analyses, IBM SPSS Statistics ver. 20.0 (IBM Corp., Armonk, USA) and Amos ver. 16.0 (IBM Corp.) were used.

\section{Results}

\section{Exploratory factor analysis}

After content validation, the remaining 44 items were suitable for factor analysis in terms of normal distribution, equal variance, type of scale (interval scale), and mutual independence. Bartlett's test resulted in $\chi^{2}=$ 11,325.77 $(\mathrm{p}<0.001)$ and the Kaiser-Meyer-Olkin measure of sampling adequacy was 0.94 , indicating the data were fit for factor analysis. The results of the exploratory factor analysis are shown in Table 1. Seven factors were extracted from the data and factor loadings of the items ranged between 0.40 and 0.89 .

We conducted iterative discussion on three items that

Table 1. Attributes Grouped Together in the Exploratory Factor Analysis for 44 Items

\begin{tabular}{|c|c|c|c|c|c|c|c|}
\hline & & & & racted fact & & & \\
\hline & Factor 1 & Factor 2 & Factor 3 & Factor 4 & Factor 5 & Factor 6 & Factor 7 \\
\hline Item 19 & 0.738 & & & & & & \\
\hline Item 18 & 0.708 & & & & & & \\
\hline Item 17 & 0.692 & & & & & & \\
\hline Item 22 & 0.675 & & & & & & \\
\hline Item 20 & 0.673 & & & & & & \\
\hline Item 23 & 0.639 & & & & & & \\
\hline Item 15 & 0.635 & & & & & & \\
\hline Item 24 & 0.608 & & & & & & \\
\hline Item 16 & 0.592 & & & & & & \\
\hline Item 26 & 0.557 & & & & & & \\
\hline Item 21 & 0.552 & & & & & & \\
\hline Item 25 & 0.530 & & & & & & \\
\hline Item $30^{\text {al }}$ & 0.430 & & & & & & \\
\hline Item 40 & & 0.826 & & & & & \\
\hline Item 37 & & 0.825 & & & & & \\
\hline Item 38 & & 0.823 & & & & & \\
\hline Item 39 & & 0.773 & & & & & \\
\hline Item 41 & & 0.699 & & & & & \\
\hline Item 34 & & 0.691 & & & & & \\
\hline Item 35 & & 0.679 & & & & & \\
\hline Item 33 & & 0.671 & & & & & \\
\hline Item 36 & & 0.629 & & & & & \\
\hline Item 11 & & & 0.808 & & & & \\
\hline Item 12 & & & 0.765 & & & & \\
\hline Item 10 & & & 0.712 & & & & \\
\hline Item 13 & & & 0.511 & & & & \\
\hline Item 8 & & & & 0.889 & & & \\
\hline Item 7 & & & & 0.789 & & & \\
\hline Item 9 & & & & 0.740 & & & \\
\hline Item $14^{\mathrm{al}}$ & & & & 0.436 & & & \\
\hline
\end{tabular}


Table 1. (Continued)

\begin{tabular}{|c|c|c|c|c|c|c|c|}
\hline & \multicolumn{7}{|c|}{ Extracted factors } \\
\hline & Factor 1 & Factor 2 & Factor 3 & Factor 4 & Factor 5 & Factor 6 & Factor 7 \\
\hline Item 2 & & & & & 0.679 & & \\
\hline Item 3 & & & & & 0.617 & & \\
\hline Item 5 & & & & & 0.600 & & \\
\hline Item 1 & & & & & 0.564 & & \\
\hline Item $6^{\text {a) }}$ & & & & & 0.477 & & \\
\hline Item $4^{\text {a) }}$ & & & & & 0.419 & & \\
\hline Item 29 & & & & & & 0.667 & \\
\hline Item 27 & & & & & & 0.533 & \\
\hline Item 28 & & & & & & 0.531 & \\
\hline Item 32 & & & & & & 0.499 & \\
\hline Item 31 & & & & & & 0.467 & \\
\hline Item 42 & & & & & & & 0.769 \\
\hline Item 44 & & & & & & & 0.697 \\
\hline Item 43 & & & & & & & 0.694 \\
\hline Eigenvalue & 7.53 & 7.51 & 3.34 & 3.32 & 3.01 & 2.68 & 2.63 \\
\hline Percentage of explained variance & 17.10 & 17.08 & 7.58 & 7.55 & 6.84 & 6.08 & 5.97 \\
\hline
\end{tabular}

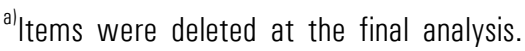

had factor loadings of less than 0.45: (1) "forcing junior residents or students to attend classes as their substitutes, for master's or doctoral degree programs"; (2) "absence from work without prior notice to clinical team"; and (3) "insisting on doctors' opinions regardless of patients'/ caregivers' preferences." Among the three items, we deleted "substitute attendance" from the questionnaire because we regarded it as a situation that was specific to the authors' institution, which requires mandatory attendance for some graduate courses. The second and third items were also removed because the authors could not reach an agreement regarding whether those behaviors were widely tolerated or only allowed in specific situations. One additional item (Item 6 in Table 1), "drinking that leads to residents having trouble working on the following day," was removed from the inventory. Although its factor loading value was above 0.45 , this item was not relevant to other items that were categorized within the same factor, that is, "misconduct lacking in respect for medical colleagues." As a result, a 40-item questionnaire under seven categories was constructed.

\section{Confirmatory factor analysis}

Following the iterative and intensive discussion of the questionnaire, we decided that it was preferable to divide the 12 items in Factor 1 into two categories according to their content relevance: professionalism lapse related to medical practice (Category 1) and behaviors in contravention to patient respect and confidentiality (Category 7). As a result, we re-categorized the 40 items into eight factors (Table 2) and named them as follows: (1) dishonesty and unsafe practice in patient care, (2) conflicts of interest, (3) misconduct in research and publication, (4) irresponsible conduct while on duty, (5) physician impairment, (6) lack of respect for colleagues, (7) disrespectful to patients and breaching confidentiality, and (8) misconduct in authorship.

A structural equation model was used for the confirmatory factor analysis of 40 items under eight 
Table 2. An Instrument Assessing Residents' Attitudes towards Professionalism Lapses (40 Items)

\begin{tabular}{|c|c|}
\hline Factor & Item $^{\text {al }}$ \\
\hline $\begin{array}{l}\text { 1. Dishonesty and unsafe } \\
\text { practice in patient care }\end{array}$ & $\begin{array}{l}\text { 1. Charting unexamined physical findings } \\
\text { 2. Neglect in improving clinical knowledge and skills } \\
\text { 3. Practicing medicine beyond one's capability on a voluntary basis } \\
\text { 4. Practicing medicine beyond one's capability as ordered } \\
\text { 5. Providing incorrect information to patients to conceal one's ignorance } \\
\text { 6. Prescribing patients with unnecessary medication for doctor's benefit } \\
\text { 7. Not reporting patient's medical condition to supervisor to avoid blame } \\
\text { 8. Concealing one's medical error } \\
\text { 9. Overlooking colleagues' medical errors }\end{array}$ \\
\hline 2. Conflicts of interest & $\begin{array}{l}\text { 10. Taking money or gifts from patients } \\
\text { 11. Conferring benefits to patients after taking money or gifts } \\
\text { 12. Having private relationship with patients }\end{array}$ \\
\hline $\begin{array}{l}\text { 3. Misconduct in research } \\
\text { and publication }\end{array}$ & $\begin{array}{l}\text { 13. Citing articles without referencing } \\
\text { 14. Combining passages from the Internet into an article } \\
\text { 15. Purchasing an article } \\
\text { 16. Referencing unread articles } \\
\text { 17. Fabricating whole data } \\
\text { 18. Fabricating partial data } \\
\text { 19. Altering data } \\
\text { 20. Falsifying statistical analysis process } \\
\text { 21. Submitting the same paper to different journals after modifying some details }\end{array}$ \\
\hline $\begin{array}{l}\text { 4. Irresponsible conduct } \\
\text { while on duty }\end{array}$ & $\begin{array}{l}\text { 22. Being late for work } \\
\text { 23. Sneaking out of hospital while on duty } \\
\text { 24. Sneaking out of hospital while on call } \\
\text { 25. Leaving work without completing hand over }\end{array}$ \\
\hline 5. Physician impairment & $\begin{array}{l}\text { 26. Use of illegal drugs } \\
\text { 27. Prescribing illegal drugs } \\
\text { 28. Inappropriate physical contact during physical examination }\end{array}$ \\
\hline $\begin{array}{l}\text { 6. Disrespectful behavior } \\
\text { to colleagues }\end{array}$ & $\begin{array}{l}\text { 29. Ignoring opinion of other healthcare professionals } \\
\text { 30. Treating other healthcare professionals in an abusive manner } \\
\text { 31. Criticizing colleague doctors or other healthcare professionals in front of patients } \\
\text { 32. Verbally or physically abusing junior doctors or students }\end{array}$ \\
\hline $\begin{array}{l}\text { 7. Being disrespectful to } \\
\text { patients and breaching } \\
\text { confidentiality }\end{array}$ & $\begin{array}{l}\text { 33. Talking about patients in public places } \\
\text { 34. Talking about patients in personal spaces or on the internet } \\
\text { 35. Leaking patients' medical information } \\
\text { 36. Humiliating patients with rude words } \\
\text { 37. Talking about patients with colleagues for fun, or slandering patients }\end{array}$ \\
\hline $\begin{array}{l}\text { 8. Misconduct in } \\
\text { authorship }\end{array}$ & $\begin{array}{l}\text { 38. Including a colleague who did not contribute to the paper as an author } \\
\text { 39. Including a colleague who did not contribute to the paper as an author, due to pressure from others } \\
\text { 40. Requesting one's own inclusion as an author despite not contributing to the paper }\end{array}$ \\
\hline
\end{tabular}

${ }^{\text {al }}$-Point Likert scale from 1 (absolutely cannot be done), 2 (cannot be done), 3 (can be done depending on circumstances,), to 4 (usually can be done).

categories (Fig. 2). As a result of the analysis, the $\chi^{2} \quad$ instrument.

was significant $(p<0.01)$, and the RMSEA and CFI were

0.07 and 0.9 , respectively, representing good fit for the 
Fig. 2. Measurement Model for an Instrument to Assess Residents' Attitudes towards Professionalism Lapses

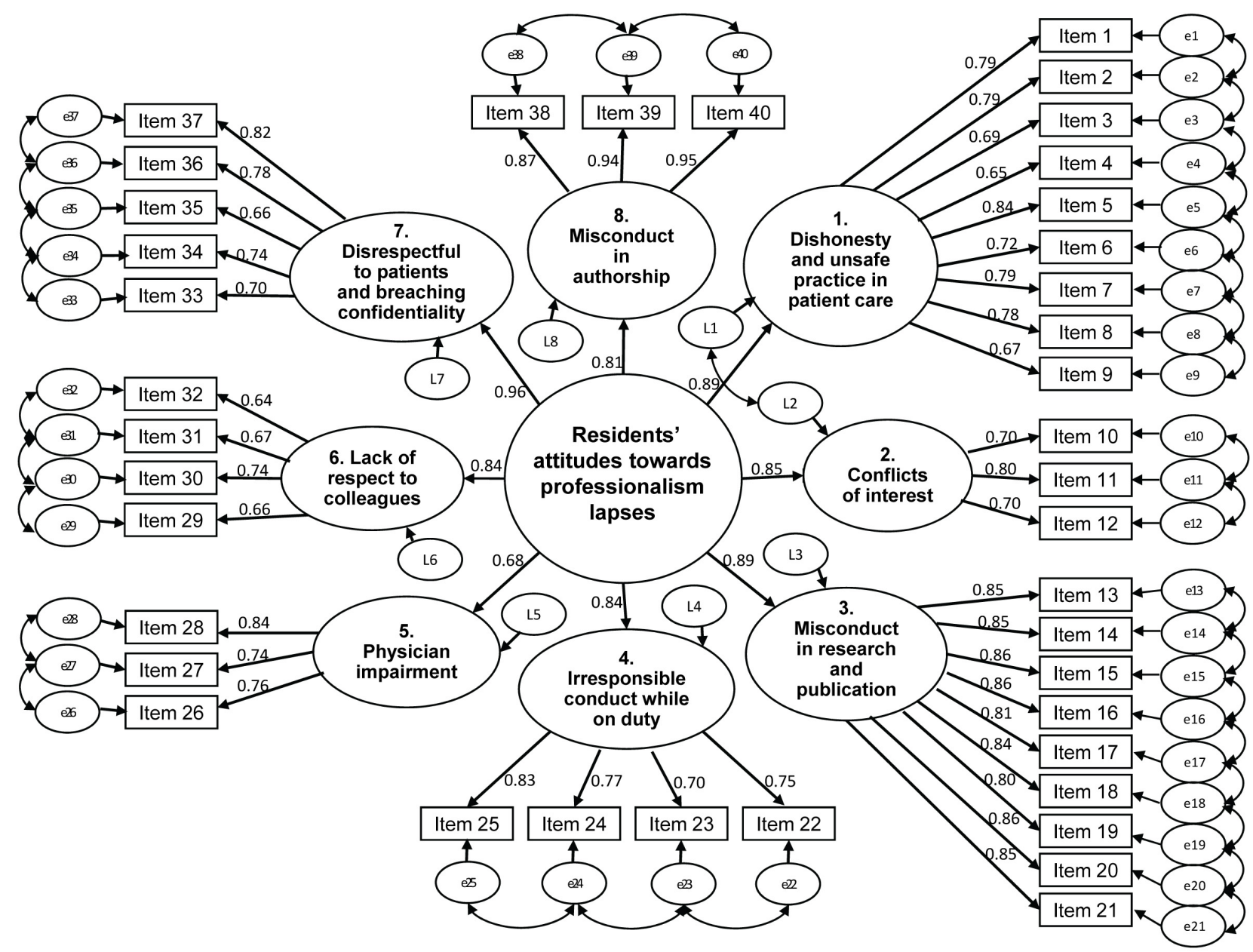

Item numbers are exactly same with those of the Table 2. The detailed information of each item is shown in Table 2.

Table 3. Reliability Coefficients of Total Items and Each Category

\begin{tabular}{lc}
\hline \multicolumn{1}{c}{ Category } & Reliability \\
\hline Total item: Residents' attitudes toward unprofessional conduct & 0.97 \\
Factor 1: Dishonesty and unsafe practice in patient care & 0.92 \\
Factor 2: Conflicts of interest & 0.76 \\
Factor 3: Misconduct in research and publication & 0.95 \\
Factor 4: Irresponsible conduct while on duty & 0.87 \\
Factor 5: Physician impairment & 0.86 \\
Factor 6: Lack of respect to colleagues & 0.75 \\
Factor 7: Disrespectful to patients and breaching confidentiality & 0.86 \\
Factor 8: Misconduct in authorship & 0.93 \\
\hline
\end{tabular}

\section{Assessment of reliability}

The Cronbach's $\alpha$ for the questionnaire was 0.97 , and it ranged from 0.75 to 0.97 for each category. The reliability coefficients of all the items and each category are shown in Table 3. 


\section{Discussion}

We developed an inventory assessing residents' attitudes toward unprofessional conduct based on the assumption that residents' perceptions of unethical behaviors or misconduct may reflect their level of standards of professionalism. The 40-item inventory was developed and validated using exploratory and confirmatory factor analysis. Because we could not find any validated tools assessing residents' professionalism in our culture, we identified latent variables through exploratory factor analysis and categorized them into eight factors. We then constructed the structural model and verified it using confirmatory factor analysis. As a result, the fit indices of the model were acceptable, as was the reliability of the entire questionnaire and its factors.

Residency is a crucial period for the development of professional identity as doctors, as residents fully engage in performing various tasks in the real world. The level of a resident's professionalism can influence their clinical performance, patient safety, and quality of patient care. In a previous study, a resident group with a low level of professionalism was reported to have a high risk of disciplinary action [8]. Therefore, previous studies have suggested the importance of residents' professionalism and methods for its enhancement $[9,10,11,18]$.

This study is distinct from previous studies in a number of ways. First, our instrument includes more comprehensive domains of behavior that may occur in the actual training environment. Most previous papers investigated unprofessional behaviors in specific circumstances $[10,18,19]$ or domains, such as alcohol and other substance abuse [20]. However, our inventory encompassed a wide range of types of resident misconduct, including patient care, respect for patients and colleagues, responsibility for work, physician impair- ment, and research and publication.

Second, we tried to incorporate some specific features originating from different sociocultural backgrounds into the inventory: for example, Item 11 ("Conferring benefits on patients after accepting money or gifts") has rarely been reported by previous studies from Western cultures. One of the distinctive features of this study was the gifted authorship who did not contribute to the paper as an author. Publication ethics, misrepresentation, plagiarism, and falsification were frequently identified resident misconducts in previous Western studies [19,21], whereas gifted authorship has rarely been reported. More interestingly, residents demonstrated a relatively tolerant attitude toward gifted authorship. A cross-cultural study on medical professionalism between Taiwan and Canada reported the prominence of Confucian relationalism and recommended further cross-cultural studies on medical professionalism to move the field beyond a Western individualist perspective [12,13]. In this study, the authors regarded Korea as one of the cultures rooted in Confucianism, in which relationalism is more pronounced. This is a distinctive feature of Korean culture. As such, we would like to raise these authorship issues for educational purposes.

We expected that the mere action of completing this questionnaire would provide residents with an opportunity for self-reflection on the unprofessional behaviors described in the inventory. Furthermore, we expect that this tool may help to identify the level of residents' personal standards for professionalism, and the areas to which medical educators should pay attention in order to improve residents' attitudes toward professionalism. Our inventory can be used to enhance residents' abilities in assessing and reflecting upon their professionalism.

Our study has several limitations. First, we developed the questionnaire items based on real-life practice situations faced by residents; however, it was impossible to 
cover all aspects of practice. Second, sample size may be not adequate for generalization; we will conduct the survey about more cases. Finally, although we did our best to define and identify unprofessional or undesirable behaviors, establishing a clear definition of unprofessional behaviors was challenging. Furthermore, we must recognize that the trend of educational research on medical professionalism is moving from the evaluation of unprofessional behavior to that of professional behavior [8]. Although this study has several limitations, the authors conclude that our inventory was a timely attempt to explore residents' attitudes and level of awareness of medical professionalism, in considering Korean medical society where studies on medical professionalism have only just commenced. Furthermore, we hope this questionnaire instrument can increase academic interest in developing more valid and reliable tools for assessing medical professionalism.

In conclusion, the authors suggest that the inventory of this study may contribute to identifying residents' perceptions and levels of consciousness on the seriousness of each type of unprofessional behavior and to define the areas of professionalism that require educational reinforcement at both individual and institutional levels.

\section{ORCID:}

Hyo-Jin Kwon: http://orcid.org/0000-0001-5238-1931; Young-Mee Lee: http://orcid.org/0000-0002-4685-9465; Young-Hee Lee: http://orcid.org/0000-0002-4584-9070; Hyung-Joo Chang: http://orcid.org/0000-0003-4554-5074

Acknowledgements: None.

Funding: This research was supported by the Basic Science Research program through the National Research Foundation of Korea (NRF), which is funded by the Ministry of Education, Science and Technology (No. 2012R1A1A
3013486).

Conflicts of interest: None.

\section{References}

1. Papadakis MA, Paauw DS, Hafferty FW, Shapiro J, Byyny RL; Alpha Omega Alpha Honor Medical Society Think Tank. Perspective: the education community must develop best practices informed by evidence-based research to remediate lapses of professionalism. Acad Med 2012; 87: 1694-1698.

2. Wynia MK, Papadakis MA, Sullivan WM, Hafferty FW. More than a list of values and desired behaviors: a foundational understanding of medical professionalism. Acad Med 2014; 89: 712-714.

3. Hodges BD, Ginsburg S, Cruess R, Cruess S, Delport R, Hafferty F, Ho MJ, Holmboe E, Holtman M, Ohbu S, Rees C, Ten Cate O, Tsugawa Y, Van Mook W, Wass V, Wilkinson T, Wade W. Assessment of professionalism: recommendations from the Ottawa 2010 Conference. Med Teach 2011; 33: 354-363.

4. Wilkinson TJ, Wade WB, Knock LD. A blueprint to assess professionalism: results of a systematic review. Acad Med 2009; 84: 551-558.

5. Cohen JJ. Professionalism in medical education, an American perspective: from evidence to accountability. Med Educ 2006; 40: 607-617.

6. Papadakis MA, Loeser H, Healy K. Early detection and evaluation of professionalism deficiencies in medical students: one school's approach. Acad Med 2001; 76: 1100-1106.

7. Teherani A, O'Sullivan PS, Lovett M, Hauer KE. Categorization of unprofessional behaviours identified during administration of and remediation after a comprehensive clinical performance examination using a validated professionalism framework. Med Teach 2009; 
31: 1007-1012.

8. Stern DT, ed. Measuring medical professionalism. New York, USA: Oxford University Press; 2006.

9. Papadakis MA, Arnold GK, Blank LL, Holmboe ES, Lipner RS. Performance during internal medicine residency training and subsequent disciplinary action by state licensing boards. Ann Intern Med 2008; 148: 869-876.

10. Fargen KM, Drolet BC, Philibert I. Unprofessional behaviors among tomorrow's physicians: review of the literature with a focus on risk factors, temporal trends, and future directions. Acad Med 2016; 91: 858-864.

11. Reed DA, West CP, Mueller PS, Ficalora RD, Engstler GJ, Beckman TJ. Behaviors of highly professional resident physicians. JAMA 2008; 300: 1326-1333.

12. Ho MJ, Lin CW, Chiu YT, Lingard L, Ginsburg S. A cross-cultural study of students' approaches to professional dilemmas: sticks or ripples. Med Educ 2012; 46: 245-256.

13. Cruess SR, Cruess RL, Steinert Y. Teaching professionalism across cultural and national borders: lessons learned from an AMEE workshop. Med Teach 2010; 32: 371-374.

14. Swick HM. Toward a normative definition of medical professionalism. Acad Med 2000; 75: 612-616.
15. Cruess SR, Johnston S, Cruess RL. "Profession": a working definition for medical educators. Teach Learn Med 2004; 16: 74-76.

16. Wynia MK. The role of professionalism and selfregulation in detecting impaired or incompetent physicians. JAMA 2010; 304: 210-212.

17. Chang HJ, Lee YM, Lee YH, Kwon HJ. Investigation of unethical and unprofessional behavior in Korean residency training. Teach Learn Med 2015; 27: 370-378.

18. Nagler A, Andolsek K, Rudd M, Sloane R, Musick D, Basnight L. The professionalism disconnect: do entering residents identify yet participate in unprofessional behaviors? BMC Med Educ 2014; 14: 60.

19. Kaley JR, Bornhorst J, Wiggins M, Yared M. Prevalence and types of misrepresentation of publication record by pathology residency applicants. Arch Pathol Lab Med 2013; 137: 979-982.

20. Aach RD, Girard DE, Humphrey H, McCue JD, Reuben DB, Smith JW, Wallenstein L, Ginsburg J. Alcohol and other substance abuse and impairment among physicians in residency training. Ann Intern Med 1992; 116: 245254.

21. Chung CK, Hernandez-Boussard T, Lee GK. "Phantom" publications among plastic surgery residency applicants. Ann Plast Surg 2012; 68: 391-395. 